Reprod. Nutr. Dévelop., 1982, 22 (6), 1073-1081.

\title{
Influence de l'alimentation minérale de la truie sur le taux de masculinité à la naissance
}

\author{
G. BOLET, L. GUÉGUEN $(*)$, P. DANDO $\left({ }^{* *}\right)$, L. OLLIVIER
}

Station de Génétique quantitative et appliquée.

(*) Station de Recherches de Nutrition, I.N.R.A., 78350 Jouy-en-Josas, France.

(**) Domaine expérimental de Bourges, I.N.R.A., Avord 18800 Baugy.

Summary. Effect on sex ratio of mineral diet in sows.

This paper reports a study on sows to check the theory of Stolkowski which hypothesizes that mineral imbalance in the diet of the female before fertilization affects the sex ratio of the progeny. Relative excess of $\mathrm{Na}^{+}$and $\mathrm{K}^{+}$ions (NaK diet) would favour the birth of males, while relative excess of $\mathrm{Ca}^{2+}$ and $\mathrm{Mg}^{2+}$ ions (CaMg diet) would favour the birth of females. We carried out two successive experiments. In the first, sows were given the diet for about 34 days, the molar $\mathrm{Na}+\mathrm{K} / \mathrm{Ca}+\mathrm{Mg}$ ratios being 2.1 for the $\mathrm{NaK}$ diet and 0.7 for the CaMg diet. Out of a total of 677 births, the sex ratio was 55.7 with the NaK diet and 48.3 with the CaMg diet. In the second experiment, the sows were treated for about 53 days and the molar ratios were 3.9 for the $\mathrm{NaK}$ diet and 0.5 for the $\mathrm{CaMg}$ diet. Out of a total of 869 piglets, the sex ratios were 50 and 53.5, respectively. Thus, the difference observed in experiment $1(0.05<P<0.10)$, which agrees with the above mentioned theory, was reversed in experiment 2 , the diet-experiment interaction being significant $(P<0.05)$. Overall, the diet did not affect the sex ratio ; the contradictory results of the two experiments showed no reproducible effect of dietary mineral imbalance on the sex ratio in pigs. There was also a significant diet-experiment interaction $(P<0.05)$ as to litter size, and a nearly significant negative relationship between sex ratio and litter size was observed.

\section{Introduction.}

II est bien connu que le taux de masculinité à la naissance (TM $=$ nombre de naissances mâles pour 100 naissances) est relativement constant et voisin de 51,5 chez les Mammifères (Courot, 1975). De très nombreuses tentatives ont été faites pour modifier ce taux, soit par des méthodes anciennes et empiriques, souvent fantaisistes, soit par des méthodes scientifiques visant à assurer une fécondation préférentielle de l'ovule par des spermatozoïdes porteurs de chromosomes $X$ ou $Y$ (Beatty, 1970 ; Schwartz, 1975). Parmi ces dernières méthodes, les diverses techniques de séparation in vitro des spermatozoïdes porteurs des chromosomes $X$ ou $Y$, basées sur des différences physico-chimiques (taille, poids, charge électrique, mobilité, teneur en ADN...) ont semblé prometteuses. 
Plusieurs tentatives ont été faites, notamment dans l'espèce bovine, par centrifugation, sédimentation, électrophorèse, échange d'ions ou tamisage moléculaire. Cependant, les résultats obtenus ne sont pas toujours reproductibles et sont souvent décevants (Courot et Esnault, 1973; Courot, 1981). D'autres méthodes reposent sur la meilleure mobilité et la plus grande fragilité du spermatozoïde porteur du chromosome $Y$. II serait ainsi possible d'augmenter le TM par insémination artificielle en limitant le décalage entre insémination et ovulation ou de le diminuer en congelant le sperme à basse température (Mortimer et Richardson, 1982).

Enfin, on peut également envisager de modifier l'environnement ou les caractéristiques de l'ovule pour favoriser la sélection des spermatozoïdes dans le tractus génital ou la fécondation préférentielle par l'un ou l'ạutre type de spermatozoïdes. C'est la base des diverses méthodes d'intervention nutritionnelle sur la femelle avant la fécondation, principalement basées sur la nutrition minérale. Egalement pressentie par Lyster (1972), l'influence de l'alimentation minérale sur le TM des Mammifères a fait l'objet de nombreuses publications depuis les premiers travaux de Stolkowski portant sur un batracien, Discoglossus pictus (OTTH), publiés en 1970. Plusieurs enquêtes rétrospectives et études prospectives dans l'espèce bovine (Stolkowski, 1967 ; Stolkowski et Emmerich, 1971 ; Stolkowski et Lefèvre, 1977) et dans l'espèce humaine (Stolkowski et Duc, 1977 ; Stolkowski et Lorrain, 1980 ; Stolkowski et Choukroun, 1981) ont conduit Stolkowski à proposer une théorie sur le rôle des ions dans la sélection du spermatozoïde porteur de $X$ ou $Y$ assurant la fécondation : un excès relatif d'ions alcalins $\left(\mathrm{Na}^{+}, \mathrm{K}^{+}\right)$favoriserait la naissance de mâles, tandis qu'un excès relatif $\mathrm{d}^{\prime}$ ions alcalino-terreux $\left(\mathrm{Ca}^{2+}, \mathrm{Mg}^{2+}\right)$, parfois associé à un apport de vitamine $\mathrm{D}$, favoriserait la naissance de femelles. Des méthodes pratiques de sélection du sexe, par déséquilibre minéral alimentaire avant la conception, ont été préconisées dans l'espèce humaine et chez les bovins.

Malheureusement, les principales données qui sont en faveur de cette théorie ont été obtenues sur deux espèces qui, de par leur faible prolificité et la grande variété de leur alimentation, se prêtent mal à une expérimentation rigoureuse. C'est pourquoi il nous a semblé utile de vérifier cette théorie sur la Truie, dont la prolificité naturelle permet d'obtenir rapidement un grand nombre de naissances et dont le régime alimentaire peut être rigoureusement contrôlé. Une première étude sur la Truie n'a pas permis à Lougnon et Picard (1982) de conclure à un quelconque effet de l'alimentation minérale sur le TM. Cependant, le déséquilibre minéral du régime utilisé dans cette expérience était trop modéré pour permettre d'espérer une modification importante. Nous avons donc réalisé deux expériences successives sur truies, la seconde expérience ayant pour but de vérifier la tendance observée dans la première, en augmentant la durée du traitement et en accentuant le déséquilibre minéral des régimes.

\section{Matériel et méthodes.}

Régimes alimentaires.

1re expérience: Dans cette première expérience, effectuée en 1980, le régime de base était le même pour les deux lots, le complément minéral étant 
seul différent. Le régime de base (Etablissements Bourassin, Cezy, Yonne) était constitué de 45 p. $100 \mathrm{~d}$ 'orge, 27 p. 100 de blé, 10 p. 100 de farine de luzerne, 6 p. 100 de tourteau de soja, 5 p. 100 de son de blé, 4 p. 100 de mélasse et 3 p. 100 de composé minéral. Les deux composés minéraux (Fabrique de Mélanges alimentaires expérimentaux, I.N.R.A., La Minière, Guyancourt, Yvelines) avaient la composition indiquée dans le tableau 1 . Un complexe vitaminique adapté aux besoins de la Truie était incorporé à l'aliment. La composition minérale des deux aliments complets est indiquée dans le tableau 3. Les rapports molaires $\mathrm{Na}+\mathrm{K} / \mathrm{Ca}+\mathrm{Mg}$ sont respectivement de 2,1 pour l'aliment riche en $\mathrm{Na}$ et $\mathrm{K}$ et de 0,7 pour l'aliment riche en $\mathrm{Ca}$ et $\mathrm{Mg}$.

\section{TABLEAU 1}

Formules des composés minéraux utilisés dans les deux expériences (p. 100)

\begin{tabular}{|c|c|c|c|c|}
\hline & \multicolumn{2}{|c|}{$1^{\text {re }}$ expérience } & \multicolumn{2}{|c|}{$2^{\mathrm{e}}$ expérience } \\
\hline & Régime NaK & Régime $\mathrm{CaMg}$ & Régime NaK & Régime $\mathrm{CaMg}$ \\
\hline $\mathrm{CaHPO}_{4}, 2 \mathrm{H}_{2} \mathrm{O}$ & 35,0 & 35,0 & 16,0 & 12,5 \\
\hline $\mathrm{CaCO}_{3}, \ldots \ldots$ & 10,0 & 48,0 & 0 & 74,0 \\
\hline $\mathrm{Mg}(\mathrm{OH})_{2}$ & 0 & 10,0 & 0 & 10,0 \\
\hline $\mathrm{KCl} \ldots .$. & 22,0 & 0 & 51,5 & 0 \\
\hline $\mathrm{NaCl} \ldots \ldots$ & 31,0 & 5,0 & 31,0 & 2,0 \\
\hline $\mathrm{ZnSO}_{4}, \mathrm{H}_{2} \mathrm{O}$. & 1,4 & 1,4 & 1,0 & 1,0 \\
\hline $\mathrm{MnSO}_{4}, \mathrm{H}_{2} \mathrm{O} \ldots \ldots$ & 0,5 & 0,5 & 0,4 & 0,4 \\
\hline $\mathrm{CuSO}_{4}, 5 \mathrm{H}_{2} \mathrm{O} \ldots$ & 0,1 & 0,1 & 0,1 & 0,1 \\
\hline
\end{tabular}

TABLEAU 2

Aliments utilisés dans la $2^{e}$ expérience (p. 100)

\begin{tabular}{|c|c|c|}
\hline & Aliment $\mathrm{NaK}$ & Aliment $\mathrm{CaMg}$ \\
\hline 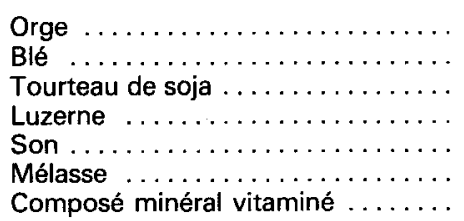 & $\begin{array}{r}44 \\
27 \\
6 \\
10 \\
5 \\
4 \\
4\end{array}$ & $\begin{array}{r}49 \\
32 \\
6 \\
0 \\
8 \\
0 \\
5\end{array}$ \\
\hline
\end{tabular}

TABLEAU 3

Composition minérale des aliments utilisés dans les deux expériences (g par $\mathrm{kg}$ )

\begin{tabular}{|c|c|c|c|c|c|c|c|c|}
\hline & \multicolumn{4}{|c|}{ 1re expérience } & \multicolumn{4}{|c|}{$2^{\mathrm{e}}$ expérience } \\
\hline & $\mathrm{Na}$ & $\mathrm{K}$ & $\mathrm{Ca}$ & $\mathrm{Mg}$ & $\mathrm{Na}$ & $k$ & $\mathrm{Ca}$ & $\mathrm{Mg}$ \\
\hline $\begin{array}{l}\text { Aliment } \mathrm{NaK} \\
\text { Aliment } \mathrm{CaMg}\end{array}$ & $\begin{array}{l}2,7 \\
0,8\end{array}$ & $\begin{array}{r}12,5 \\
9,5\end{array}$ & $\begin{array}{l}4,2 \\
8,5\end{array}$ & $\begin{array}{l}2,4 \\
4,0\end{array}$ & $\begin{array}{l}3,7 \\
0,5\end{array}$ & $\begin{array}{r}20,4 \\
9,6\end{array}$ & $\begin{array}{r}3,7 \\
15,3\end{array}$ & $\begin{array}{l}2,0 \\
3,7\end{array}$ \\
\hline
\end{tabular}


$2^{e}$ expérience : Cette nouvelle expérience a été effectuée en 1981. Dans le but de diminuer la teneur en potassium du régime CaMg, l'incorporation de luzerne et de mélasse a été évitée. Les deux aliments de base utilisés sont décrits dans le tableau 2 et les formules des deux composés minéraux correspondants figurent au tableau 1. Toutes les fabrications ont été assurées par la Fabrique de Mélanges alimentaires expérimentaux de I'I.N.R.A. Les deux régimes étaient supplémentés par $5000 \mathrm{UI}$ de vitamine $\mathrm{A}$ par $\mathrm{kg}$, le régime CaMg recevant seul un supplément de $2000 \mathrm{Ul}$ de vitamine $D_{3}$ par $\mathrm{kg}$. Toutes les autres vitamines indispensables étaient incorporées au régime de base sous la forme d'un complexe vitaminique adapté aux besoins de la Truie. La composition minérale des deux aliments complets est indiquée dans le tableau 3 . Les rapports molaires $\mathrm{Na}+\mathrm{K} / \mathrm{Ca}+\mathrm{Mg}$ sont respectivement de 3,9 pour l'aliment $\mathrm{NaK}$ et de 0,5 pour I'aliment CaMg.

\section{Matériel animal et dispositif expérimental.}

Nous avons utilisé pour la première et la deuxième expérience les truies et les verrats appartenant respectivement à la $12^{\mathrm{e}}$ et à la $13^{\mathrm{e}}$ génération d'une expérience de sélection sur la prolificité (Ollivier et Bolet, 1981), menée dans l'élevage expérimental du département de Génétique animale de I'I.N.R.A. situé à Avord (Cher). Les jeunes truies recevaient individuellement, à partir de $100 \mathrm{~kg}$ de poids vif, l'aliment expérimental ( $3 \mathrm{~kg}$ par jour) pendant environ un mois avant le premier accouplement ; le régime était ensuite poursuivi jusqu'à confirmation de la gestation. Dans l'une et I'autre expérience, 7 groupes étaient constitués, composés chacun de 10 à 15 truies saillies par un même verrat. Dans la première expérience, les truies de 4 groupes recevaient l'aliment $\mathrm{CaMg}$ et celles des 3 autres groupes l'aliment NaK ; les accouplements se sont déroulés du 15 mai au 15 août 1980. Dans la deuxième expérience, les deux aliments étaient répartis au hasard entre les truies accouplées à un même verrat ; les accouplements se sont déroulés du 1er juin au 20 septembre 1981.

\section{Méthodes d'analyse.}

- Analyse minérale. Les teneurs en éléments minéraux indiquées dans le tableau 3 ont été obtenues par analyse de plusieurs échantillons représentatifs des aliments. Après incinération au four à $530^{\circ} \mathrm{C}$ pendant 10 heures, les cendres sont mises en solution acide et, après dilution convenable, $\mathrm{Na}, \mathrm{K}$ et $\mathrm{Ca}$ sont dosés par spectrophotométrie d'émission de flamme (appareil Eppendorf) et $\mathbf{M g}$ par spectrométrie d'absorption atomique (appareil I.L. 151).

- Analyse statistique. Pour chaque truie, nous avons relevé l'âge au début du traitement, à la puberté et à la saillie fécondante, ainsi que le nombre total de porcelets de chaque sexe, nés vivants ou morts. Nous avons étudié l'effet du régime sur le TM pour chaque expérience en calculant le $\chi_{\mathrm{c}}^{2}$ avec la correction de continuité (Snedecor et Cochran, 1980) et, sur les deux années, en calculant le $\chi_{7}^{2}$ global et le $\chi_{1}^{2}$ d'interaction expérience $x$ régime (Kendall et Stuart, 1961).

Nous avons également réalisé une analyse de variance à deux voies lexpérience et régimel, avec interaction sur la taille de portée et calculé la régression 
linéaire du TM de la portée sur la taille $(n)$ de celle-ci, chaque TM étant affecté d'une pondération égale à $n$, puisque TM est une variable aléatoire binomiale de variance proportionnelle à $1 / n$.

\section{Résultats.}

Déroulement des deux expériences. - Sur l'ensemble des deux expériences, 188 truies ont été mises à la reproduction, dont 145 ont mis bas un total de 1546 porcelets ; le taux de fertilité a été de 74,4 p. 100 et 79,4 p. 100 respectivement pour les deux expériences ; le traitement n'a pas eu d'effet significatif sur ce paramètre $\left(\chi^{2}=0\right.$ dans les deux cas $)$. Le traitement a duré en moyenne 34 jours dans la première expérience et 53 jours dans la deuxième (tabl. 4 ).

TABLEAU 4

Effectif et âge des truies aux différents stades des deux expériences

\begin{tabular}{|c|c|c|}
\hline Expérience & 1 & 2 \\
\hline $\begin{array}{l}\text { Nombre de truies mises à la reproduction } \ldots \ldots \ldots \ldots \ldots \ldots \ldots \ldots \ldots \\
\text { Nombre de gestations } \ldots \ldots \ldots \ldots \ldots \ldots \ldots\end{array}$ & $\begin{array}{l}86 \\
64\end{array}$ & $\begin{array}{r}102 \\
81\end{array}$ \\
\hline 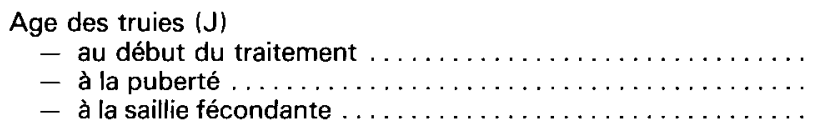 & $\begin{array}{l}256 \pm 2 \\
274 \pm 3 \\
290 \pm 3\end{array}$ & $\begin{array}{l}244 \pm 2 \\
269 \pm 3 \\
297 \pm 3\end{array}$ \\
\hline 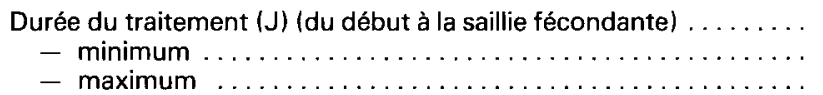 & $\begin{array}{l}34 \pm 2 \\
13 \\
83\end{array}$ & $\begin{array}{r}53 \pm 2 \\
29 \\
138\end{array}$ \\
\hline
\end{tabular}

Effet des régimes expérimentaux sur le taux de masculinité (tabl. 5). Dans la $1^{\text {re }}$ expérience, le TM est plus élevé avec le régime $\operatorname{NaK}(55,7$ p. 100) qu'avec le régime $\mathrm{CaMg} \mathrm{(48,3} \mathrm{p.} \mathrm{100);} \mathrm{cette} \mathrm{différence} \mathrm{est} \mathrm{proche} \mathrm{du} \mathrm{seuil} \mathrm{de}$ signification $\left(\chi_{1 c}^{2}=3,29 ; 0,05<P<0,10\right)$. Dans la $2^{\mathrm{e}}$ expérience, le TM est plus élevé avec le régime CaMg (53,5 p. 100) qu'avec le régime NaK (50 p. 100) mais cette différence n'est pas significative $\left(\chi_{1 \mathrm{c}}^{2}=0,90\right)$. Globalement, il $n^{\prime} y$ a pas de différence significative entre les deux traitements $\left(\chi_{1}^{2}=0,21\right)$ ni entre les deux expériences $\left(\chi_{1}^{2}=0,04\right)$. Par contre, le $\chi^{2} \mathrm{~d}^{\prime}$ interaction expérience $\mathrm{x}$ régime est significatif $\left(\chi_{1}^{2}=4,1 ; p<0,05\right)$.

Effet des régimes sur la prolificité (tabl. 6). - Dans la 1'e expérience, la prolificité des truies soumises au régime CaMg est significativement plus élevée que celle des truies soumises au régime $\operatorname{NaK}(+1,5$ porcelet, $\mathrm{P}<0,05)$; dans la $2^{\mathrm{e}}$ expérience, la tendance est inversée $(-0,6)$ mais non significative. L'analyse de variance met en évidence une interaction expérience $x$ régime significative $(F=4,6 ; P<0,05)$. 
TABLEAU 5

Effet du régime alimentaire sur le taux de masculinité

\begin{tabular}{|c|c|c|c|c|c|c|}
\hline \multirow{3}{*}{ Expérience } & \multirow{3}{*}{$x_{c}^{2}$} & \multicolumn{4}{|c|}{ Traitement } & \multirow{3}{*}{$\begin{array}{c}\text { Taux } \\
\text { de masculinité } \\
\text { global } \\
(p .100)\end{array}$} \\
\hline & & \multicolumn{2}{|c|}{$\begin{array}{c}\text { CaMg } \\
\text { " femelle " }\end{array}$} & \multicolumn{2}{|c|}{$\begin{array}{c}\mathrm{NaK} \\
\text { " mâle " }\end{array}$} & \\
\hline & & $\begin{array}{c}\text { Nombre } \\
\text { de porcelets }\end{array}$ & $\begin{array}{c}\text { Taux } \\
\text { de masculinité } \\
\text { (p. 100) }\end{array}$ & $\begin{array}{c}\text { Nombre } \\
\text { de porcelets }\end{array}$ & $\begin{array}{c}\text { Taux } \\
\text { de masculinité } \\
\text { (p. 100) }\end{array}$ & \\
\hline 1 & \multirow{3}{*}{$\begin{array}{l}3,3 \\
\text { n.s. } \\
0,9 \\
\text { n.s. } \\
0,2 \\
\text { n.s. }\end{array}$} & 404 & 48,3 & 273 & 55,7 & 51,3 \\
\hline 2 & & 449 & 53,5 & 420 & 50,0 & 51,8 \\
\hline $1+2$ & & 853 & 51,0 & 693 & 52,2 & 51,6 \\
\hline
\end{tabular}

On constate que les variations de prolificité entre régimes intra-année sont essentiellement dues à des variations du nombre de femelles dans la portée (tabl. 6), significatives dans la $1^{r e}$ expérience $(+1,5 ; P<0,05)$, non significatives dans la $2^{\mathrm{e}}(-0,7)$, donc liées à des variations de TM. Le coefficient de régression linéaire du TM sur la taille de portée est de $-0,93 \pm 0,54$ (non significatif).

\section{TABLEAU 6}

Relations entre le régime alimentaire et les caractéristiques des portées (sur chaque ligne, les valeurs affectées des mêmes lettres ne sont pas significativement différentes)

\begin{tabular}{|c|c|c|c|}
\hline & Régime & $\mathrm{CaMg}$ & NaK \\
\hline $\begin{array}{c}\text { Expérience } \\
1\end{array}$ & $\begin{array}{c}\text { Nombre de mises-bas } \ldots \ldots . \\
\text { Taille de portée ............ } \\
\text { Nombre de mâles par portée. } \\
\text { Nombre de femelles par } \\
\text { portée } \ldots \ldots \ldots \ldots \ldots \ldots\end{array}$ & $\begin{array}{c}36 \\
11,22 \pm 0,47 a^{\prime} \\
5,42 \pm 0,36 a^{\prime} \\
5,81 \pm 0,44 a^{\prime \prime}\end{array}$ & $\begin{array}{l}28 \\
9,75 \pm 0,54 b \\
5,43 \pm 0,43 a^{\prime} \\
4,32 \pm 0,35 b^{\prime \prime}\end{array}$ \\
\hline $\begin{array}{l}\text { Expérience } \\
2\end{array}$ & $\begin{array}{l}\text { Nombre de mises-bas } \ldots \ldots \ldots \\
\text { Taille de portée .......... } \\
\text { Nombre de mâles par portée } \\
\text { Nombre de femelles par } \\
\text { portée } \ldots \ldots \ldots \ldots \ldots \ldots \ldots\end{array}$ & $\begin{array}{c}43 \\
10,43 \pm 0,43 a^{\prime} \\
5,58 \pm 0,31 a^{\prime} \\
4,86 \pm 0,39 a^{\prime \prime}\end{array}$ & $\begin{array}{c}38 \\
11,05 \pm 0,50 a^{\prime} \\
5,53 \pm 0,37 a^{\prime} \\
5,53 \pm 0,35 a^{\prime \prime}\end{array}$ \\
\hline
\end{tabular}

\section{Discussion.}

Ces deux expériences successives mettent en évidence des effets contradictoires de l'alimentation minérale de la Truie sur le taux de masculinité à la naissance. La faible tendance (non significative au seuil de $P=0,05$ ) observée dans la première expérience, dans le sens escompté selon les hypothèses de Stol- 
kowski (plus forte proportion de mâles avec le régime riche en $\mathrm{Na}$ et $\mathrm{K}$ ), est contredite par une tendance moins nette en sens inverse dans la seconde expérience. On peut donc essayer d'expliquer cette interaction, qui est significative, par les conditions différentes des deux expériences, mais ce sont celles de la seconde qui étaient sans doute les plus favorables à la mise en évidence d'un éventuel effet des apports minéraux. En effet, non seulement la durée du traitement était plus longue (en moyenne 19 jours de plus, aucune truie n'ayant reçu le régime expérimental pendant moins de 29 jours avant la fécondation), mais le déséquilibre minéral du régime était beaucoup plus accentué. Ainsi, les rapports molaires $\mathrm{Na}+\mathrm{K} / \mathrm{Ca}+\mathrm{Mg}$ étaient respectivement de 3,9 et de 0,5 dans les deux régimes de la deuxième expérience, ce rapport étant normalement voisin de 1 dans I'alimentation classique du porc. De plus, dans cette seconde expérience, un supplément de vitamine $D_{3}$ dans l'aliment CaMg devait permettre de valoriser au mieux I'utilisation du calcium. Par ailleurs, dans la première expérience, toutes les truies affectées à un même verrat recevaient le même aliment, ce qui ne permet pas de distinguer l'effet du régime d'un éventuel effet du verrat sur le TM de la portée qu'il engendre. Nishida et al. (1969) ont en effet mis en évidence des variations de TM entre verrats. Par contre, le dispositif expérimental de la deuxième expérience permettait d'éliminer cet éventuel effet. Toutefois, sur les deux expériences, il n'apparaît pas d'effet significatif du verrat sur le TM $\left(\chi_{17}^{2}=19,2\right)$. II $n^{\prime} y$ a donc pas d'explication claire de l'interaction expérience $x$ régime. II faut, par ailleurs, souligner que les effets des régimes sur TM sont faibles, puisque dans le "meilleur » des cas (régime NaK expérience 1), l'écart au TM attendu est seulement d'environ 4 points (tabl. 5).

Bien que la régression du TM sur la taille de portée ne soit pas significative, on ne peut écarter la possibilité d'une liaison faiblement négative entre ces deux variables. L'effet éventuel du régime sur le TM pourrait alors s'exercer à travers un effet sur la taille de portée, toutefois difficilement explicable. Les variations de TM s'expliqueraient par une variation de la mortalité embryonnaire et/ou fœtale des femelles, plus importante dans les petites portées. Kennedy et Moxley (1978) n'observent pas de relation entre TM et taille de portée ; par contre, Nishida et al. (1969, 1971, 1977) obtiennent, dans certains échantillons, des résultats inverses du nôtre.

L'existence $d$ 'interactions expérience $x$ régime significatives sur le TM et sur la taille de la portée doit susciter une grande prudence en matière d'effet principal, notamment d'effet du régime. Que l'on considère les résultats de chaque expérience prise isolément ou les résultats cumulés des deux expériences, il n'apparaît pas d'effet significatif du régime sur le TM, alors que les seuls résultats de la première expérience auraient pu nous conduire à une fausse conclusion hâtive.

Ainsi, chez la Truie, dont les conditions d'élevage permettent un contrôle absolu de l'alimentation, notamment des apports minéraux, l'inversion du rapport alcalins/alcalino-terreux ne provoque aucune modification du TM. En outre, notre expérience montre l'importance d'un nombre élevé de descendants pour pouvoir conclure. Ces deux conditions n'ont jamais pu être réunies dans les expériences conduites chez des espèces unipares. Bien entendu, il peut être 
objecté que la Truie, par la taille de sa portée, est un cas particulier qui ne répond pas à la théorie générale émise pour les espèces unipares. Enfin, le fait que les truies utilisées étaient primipares et que le traitement ait souvent commencé sur des animaux encore impubères peut aussi être invoqué. Mais il resterait alors à expliquer pourquoi un mécanisme prétendu général d'action des ions minéraux au niveau du spermatozoïde fécondant et de l'ovocyte n'agirait pas du tout dans nos conditions expérimentales, alors que les mêmes mouvements ioniques transmembranaires dans les gamètes ont été décrits dans toute la série animale au moment de la fécondation (Epel, 1977; Yanagimachi, 1981).

Reçu en septembre 1982.

Accepté en septembre 1982.

\section{Références}

BEATTY R. A., 1970. The genetics of the mammalian gamete. Biol. Rev., 45, 73-119.

COUROT M., 1975. Le choix du sexe est-il possible chez les mammifères ? In C. THIBAULT, La Fécondation, Masson éd., Paris.

COUROT M., 1981. Peut-on choisir le sexe des enfants? La Recherche, 12, 218-220.

COUROT M., ESNAULT C., 1973. Sédimentation du sperme et sex-ratio chez les bovins. Ann. Biol, anim. Bioch. Biophys., 13, 329-334.

EPEL D., 1977. The program of fertilization. Scient. Amer., 237, 128-138.

KENDALL M. G., STUART A., 1961. The advanced theory of statistics, vol, II, p. 580, Ch. Griffin ed., London.

KENNEDY B. W., MOXLEY J. E., 1978. Genetic and environmental factors influencing litter size, sex ratio and gestation length in the pig. Anim. Prod., 27, 35-42.

LOUGNON J., PICARD M., 1982. A propos du sex-ratio chez le Porc. Journées Rech. Porcine en France, I.N.R.A.-I.T.P. éd., Paris, 14, 65-74.

LYSTER W. R., 1972. The sex ratios of human and sheep births in areas of high mineralisation. Intern. J. environm. Studies, 2, 309-316.

MORTIMER D., RICHARDSON D. W., 1982. Sex ratio of births resulting from artificial insemination. Br. J. Obstet. Gynaecol., 89, 132-135.

NISHIDA S., MAMBA K., SETA S., OTSUKA J., SHUDO S., TOKORO K., 1969. Sex ratio of offspring in domestic animals: Swine (1). Jap. J. zootech. Sci., 40, 449-462.

NISHIDA S., OTSUKA J., SAITO K., 1971. Sex ratio of offspring in domestic animals: Swine (2). Jap. J. zootech. Sci, 42, 71-78.

NISHIDA S., OTSUKA J., YAMAGISHI T., SUGAYA T., 1977. Sex ratio of offspring in domestic animals : Swine (7). Jap. J. anim. Reprod., 23, 55-59.

OLLIVIER L., BOLET G., 1981. La sélection sur la prolificité chez le Porc : Résultats d'une expérience de sélection sur dix générations. Journées Rech. Porcine en France, I.N.R.A.-I.T.P. éd., Paris, 13, 261-268.

SCHWARTZ D., 1975. Le choix du sexe est-il possible chez l'homme? Faits et hypothèses. In C. THIBAULT, La Fécondation, Masson éd., Paris.

SNEDECOR G. W., COCHRAN W. G., 1980. Statistical methods, 7e ed., lowa State Univ. Press ed., USA.

STOLKOWSKI J., 1967. Influence possible de la nutrition minérale sur la répartition des sexes chez la Vache : une enquête rétrospective. C. R. Acad. Sci., Paris, 265, 1059-1062.

STOLKOWSKI J., 1970. Le déterminisme ionique de la formation du sexe, étudié chez un batracien anoure: Discoglossus pictus (OTTH), et dans le règne animal. Biol. Méd., 59, 289-405. 
STOLKOWSKI J., 1977. Le magnésium dans la reproduction animale et chez l'homme. Rev. can. Biol., 36, 135-177.

STOLKOWSKI J., CHOUKROUN J., 1981. Preconception selection of sex in man. /sr. J. medic. Sci., 17, 1061-1067.

STOLKOWSKI J., DUC M., 1977. Alimentation minérale $\left(\mathrm{Na}^{+}, \mathrm{K}^{+}, \mathrm{Ca}^{2+}, \mathrm{Mg}^{2+}\right)$ chez des femmes n'ayant que des enfants du même sexe. Enquête rétrospective. Cah. Nutr. Diét., 12, $153-156$.

STOLKOWSKI J., EMMERICH E., 1971. Influence de la nutrition minérale de la Vache sur la répartition des sexes dans la descendance : une enquête prospective, un essai expérimental. Ann. Endocr., 32, 3-15.

STOLKOWSKI J., LEFEVRE M., 1977. Essais de contrôle du sexe chez les bovins sous l'influence de la nutrition minérale. Rec. Méd. vét., 153, 37-44.

STOLKOWSKI J., LORRAIN J., 1980. Preconceptional selection of fetal sex. Int. J. Gynaecol. Obstet., 18, 440-443.

YANAGIMACHI R., 1981. Mechanisms of fertilization in mammals. In MASTROIANNI L. Jr. et BIGGERS J. D., Fertilization and embryonic development in vitro, Plenum Publ. Corp. 